\title{
Inhaler Review in Older Adults with Asthma or COPD: A Cost-Effectiveness Study and a Perspective in Portugal
}

\author{
Tiago Maricoto, MD, *广 (1) João Marques-Gomes, PhD, Jaime Correia-de-Sousa, PhD, ${ }^{\S \Phi l}$ and \\ Luís Taborda-Barata, $\mathrm{PhD}^{/ * *}$
}

OBJECTIVES: Older patients with asthma or chronic obstructive pulmonary disease are particularly susceptible to exacerbations that may be associated with incorrect use of inhalers. Educational programs with inhaler technique review seem to be effective, but no studies have addressed their cost-effectiveness in older adult patients.

The objective was to perform a cost-effectiveness analysis of education programs in older patients and estimate the cost benefit of applying such a program in Portugal.

DESIGN: We developed a decision tree analysis from a healthcare perspective, according to intervention costs and the exacerbation rates and costs described in a previous metaanalysis. A sensitivity analysis of worst and best case scenarios was performed to estimate thresholds for intervention affordable limits, as well as cost-saving estimations and incremental cost-effectiveness ratios (ICERs) for a Portuguese scenario.

SETTING AND PARTICIPANTS: We estimated cost-effectiveness thresholds applicable in all settings and performed a sensitivity analysis of a theoretical intervention model in all patients including an inhaler technique review at an annual appointment with a doctor and a nurse.

RESULTS: In the best case scenario, the intervention affordable budget could be up to almost $1800 €$ (US \$1585.24) per patient per year. Mean intervention-associated savings in

From the *Aveiro-Aradas Family Health Unit, Aveiro Health Centre, Aveiro, Portugal; 'Faculty of Health Sciences, University of Beira Interior, Covilhã, Portugal; ${ }^{*}$ Nova Medical School, Nova School of Business and Economics, Lisbon, Portugal; ${ }^{8}$ Life and Health Sciences Research Institute (ICVS)/3B's - PT Government Associate Laboratory, University of Minho,

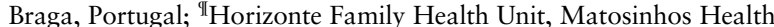
Centre, Matosinhos, Portugal; "CICS - Health Sciences Research Centre \& NuESA-Environment \& Health Study Group, Faculty of Health Sciences, University of Beira Interior, Covilhã, Portugal; and the * Department of Allergy \& Clinical Immunology, Cova da Beira University Hospital Centre, Covilhã, Portugal.

Address correspondence to Tiago Maricoto, MD, USF Aveiro-Aradas, Praceta Rainha D. Leonor, 3800, Aveiro, Portugal; Faculty of Health Sciences; University of Beira Interior, Covilhã, Portugal;

E-mail: tiago.maricoto@gmail.com

DOI: $10.1111 /$ jgs.15834
Portugal would be $311.88 €$ (US \$274.68) per patient per year, representing annual savings up to $€ 131$ million (US $\$ 150$ million) for the whole health system, already including intervention costs. ICERs for Portugal vary between 93.73€ (US \$82.55) and 437.43€ (US \$385.25) per exacerbation avoided.

CONCLUSION: A model of an intervention program with an inhaler technique review in older adult patients suggests that this intervention is cost-effective and can generate significant savings. J Am Geriatr Soc 67:1430-1436, 2019.

Key words: cost-effectiveness; inhaler; asthma; chronic obstructive pulmonary disease; older adults

$\mathrm{R}$ espiratory diseases are one of the main causes of death worldwide. Asthma and chronic obstructive pulmonary disease (COPD) affect up to $10 \%$ of the population. ${ }^{1}$ Inhaled therapy is the main treatment pathway used, but most patients use devices incorrectly, frequently with critical errors, thereby contributing to poor clinical control and increased risk of exacerbations. ${ }^{2-5}$ In addition, many studies showed that poor clinical control also leads to increased costs in health services. ${ }^{6-9}$ However, true estimates of the burden due to asthma and COPD are unavailable because direct and indirect costs are difficult to quantify, and different parts of the world report different estimations.

Significant evidence shows that educational programs for asthma and COPD may be effective in terms of clinical improvement, ${ }^{10-14}$ but few have addressed inhaler technique review alone. In addition, strong evidence supports the cost-effectiveness of those programs, although the reported results show a broad range of annual cost savings with interventions, ranging from $200 €$ (US \$176.17) to $2000 €$ (US \$1761.70) per patient. ${ }^{15-20}$ In Portugal, acute care and exacerbations-associated annual costs are estimated between 330€ (US \$290.69) and 8000€ (US \$7047.30) per patient, ${ }^{7,21}$ but no study has yet evaluated the cost-effectiveness 
of a conceptualized national education program for asthma and COPD.

Portugal has one of the oldest populations in Europe, and older adult patients tend to have a poor quality of life. ${ }^{22}$ Also, respiratory diseases represent the second leading cause of death in these patients. ${ }^{23}$ Older patients with asthma or COPD are particularly susceptible to poor disease control and exacerbations, ${ }^{24,25}$ due to particular characteristics such as increased comorbidities, poor adherence to treatment, and inadequate inhaler technique. ${ }^{26-28}$ Nevertheless, a recent meta-analysis showed that educational interventions of inhaler technique review in older patients significantly reduced the risk of exacerbations, with a pooled risk reduction of $29 \%{ }^{29}$ These are patients who may benefit most from educational interventions. This reported effectiveness was not previously described in younger patients. ${ }^{30,31}$

However, as far as we know, no cost-effectiveness study has been published that has analyzed educational interventions in older patients. Decision analysis and costeffectiveness analysis are useful tools that integrate evidence in specific context conditions to address a specific decision problem. ${ }^{32}$ Developed countries face the need to reduce health costs and maximize clinical benefits from interventions. Thus one major strategy is to identify the most costeffective subgroups of patients.

We hypothesized that a simple intervention (review of inhaler technique) would result in a slight increase in intervention costs but also in a decrease in direct costs of exacerbations, resulting in overall healthcare costs savings in a model for older patients with asthma or COPD. Thus our study aims to determine, in accordance with the main results reported in the previous meta-analysis, the costeffectiveness of educational interventions including inhaler technique review in older patients with asthma or COPD.

\section{METHODS}

\section{Study Design and Framework}

We developed a standard cost-effectiveness analysis, based on a decision tree approach ${ }^{33}$ and in accordance with CHEERS recommendations (Supplementary Appendix S1). ${ }^{34}$ Our aims were to perform estimations of treatment-affordable thresholds and according to exacerbation costs in older patients. In addition, we performed estimations of cost savings and incremental cost-effectiveness ratios (ICERs) for a Portuguese scenario, according to local costs of a theoretical intervention program.

\section{Sources of Data}

We used data from previously published studies on exacerbation costs, exacerbation rates, and local costs for intervention on inhaler technique review to determine cost-effectiveness ratios. According to a previously published meta-analysis, interventions that include inhaler technique review in older patients with asthma or COPD reduce exacerbation rates from 0.58 to 0.43 (number of exacerbations per patient per year). Absolute mean reductions of exacerbation rates range from 0.07 to 0.22 , in worst and best case scenarios and according to $95 \%$ confidence interval (CI) limits. ${ }^{29}$ In addition, exacerbations and acute medical care, alone, represent annual costs between $330 €$ (US \$290.69) and $8000 €$ (US \$7047.30) per patient. ${ }^{7,21}$ A more recent estimation for COPD in Portugal points out annual costs of $2250 €$ (US \$1982.04) per patient. ${ }^{35}$ Due to the wide range of values, we used all these references to estimate scenarios for the best case, worst case, and mean estimation.

\section{Base-Case Definition}

The base-case population of our analysis were older patients with asthma or COPD because these patients are more susceptible to poor clinical control and exacerbation risks. This is a healthcare payer's perspective study in which only direct costs were considered because indirect costs and each patient's own costs were not reliably available. We did not consider medication costs in the different stages of disease management either. Two types of costs were calculated, the intervention costs and the exacerbation costs, which were assigned to the decision tree analysis. This model assumes the previously reported exacerbation risk reduction, as well as the costs associated with each exacerbation. We also performed sensitivity analysis to assess uncertainty regarding thresholds of intervention cost-effectiveness, using reported 95\% CI limits to estimate worst and best case scenarios.

\section{Intervention}

Different intervention programs were tested in these patients in previous studies, with a wide variety of aspects addressed. Most interventions addressed inhaler technique review, selfmanagement tools, and functional control, and almost all studies performed it only at baseline of the follow-up period. In addition, interventions were delivered by different health professionals such as doctors, nurses, and pharmacists. ${ }^{29}$ For that reason, we developed a conceptual intervention program with annual control appointments by a doctor and a nurse. Each appointment would require a 20-minute evaluation to perform inhaler technique review and to assess clinical control and lung function through spirometry.

\section{Setting}

Due to inherent difficulties in specifying different aspects of interventions (inhaler technique review, self-management strategies, lung function evaluation, etc), the costs of such programs were calculated as a whole, considering health professionals' salaries in 2017 based on the official values defined by the Portuguese Central Administration of Health System, as well as spirometry costs. ${ }^{7}$ All costs and outcomes were expressed as additional factors to the main comparator, which was Usual Care. We used Usual Care as the main comparator because it was the reference control reported in most studies that was included in the previous meta-analysis. ${ }^{29}$ The time frame in the base-case analysis was 1 year because exacerbation rates were reported that way. No discount rates were considered in cost estimation.

\section{Outcome Measures}

We used a synthesis-based estimate to define outcome measures that included exacerbation rates, cost per exacerbation, 


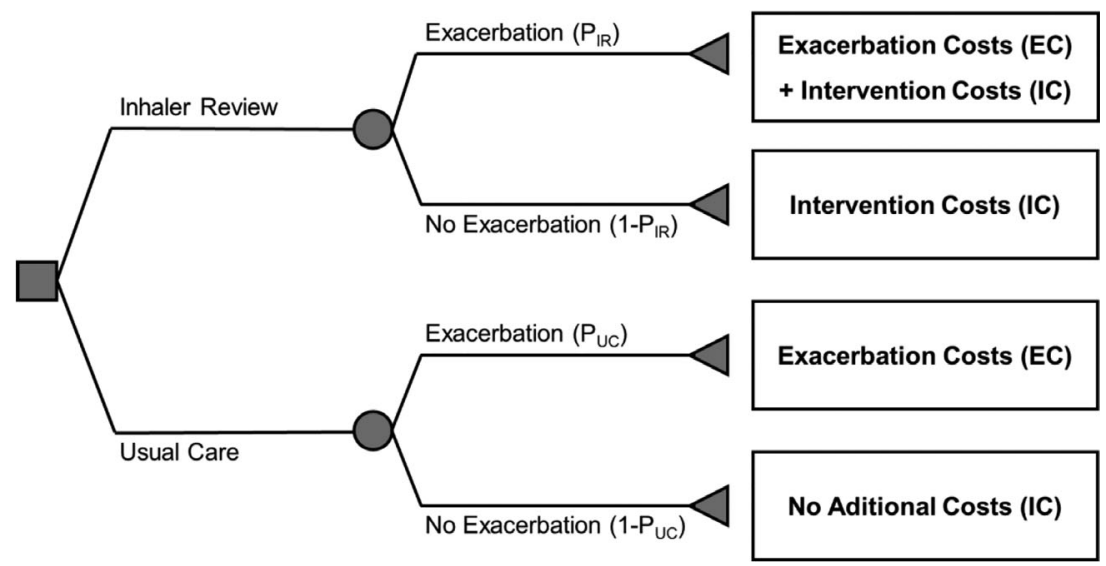

Figure 1. Decision tree model to compare Usual Care vs Inhaler technique review intervention. EC, Exacerbation costs; IC, Intervention costs; $\mathrm{P}_{\mathrm{IR}}$, Probability of exacerbation under inhaler technique review; $\mathrm{P}_{\mathrm{UC}}$, Probability of exacerbation under Usual Care.

and thresholds of cost-effectiveness for the designed intervention program. All cost estimations were used according to 2017 references, in euros $(€)$. Exacerbation costs were considered as a whole in mean estimations, regardless of the type of treatment or management that is usually provided to patients. For that reason, some types of clinical interventions provided for the management of exacerbations could be the same as those provided in our theoretical intervention program. However, that would not hamper the ability to compare them as a conceptual framework for the costeffectiveness analysis.

Figure 1 shows the decision tree used in the model. The resulting equation for cost-effectivity balance is the following one (where EC = Exacerbation costs; IC = Intervention Costs; PIR = Probability of exacerbation under Inhaler Technique Review; and PUC = Probability of exacerbation under Usual Care):

Expected Value $($ InhalerReview $)=$ Expected Value $($ Usual Care $)$ $\left[(E C+I C) \times P_{I R}\right]+\left[I C \times\left(1-P_{I R}\right)\right]=\left(E C \times P_{U C}\right)+\left[0 \times\left(1-P_{U C}\right)\right]$ $I C=E C \times\left(P_{U C}-P_{I R}\right)$
Table 1 summarizes all data considered in parameters assigned to cost estimations. Using the model assumptions presented in Figure 1, we estimated the affordable limits for intervention costs at mean values and at worst and best case scenarios. Worst case scenario was estimated using the $95 \%$ CI lower limit of probability of exacerbation under Usual Care and 95\% CI upper limit of probability of exacerbation under Inhaler Technique Review intervention. Best case scenario was estimated using the $95 \%$ CI upper limit of Probability of exacerbation under Usual Care, and 95\% CI lower limit of Probability of exacerbation under Inhaler Technique Review intervention. Mean estimation was obtained with the respective mean values.

Cost-saving estimation for Portugal was also obtained according to worst and best case scenarios. In the worst case scenario, we used the lower limit of reported exacerbation cost, the worst case estimation of intervention cost and worst case estimation of risk difference (the difference between exacerbation risk of Inhaler Technique Review group and Usual Care group). In the best case scenario, we used the upper limit of reported exacerbation cost, the best case estimation of intervention cost, and the best case estimation of risk difference.

\section{Table 1. Input parameters used in the model}

\begin{tabular}{|c|c|c|c|c|c|}
\hline \multicolumn{6}{|l|}{ Annual exacerbation rate } \\
\hline - Usual care & 0.58 & 0.5 & & 0.62 & 29 \\
\hline \multicolumn{2}{|l|}{ Parameter } & Mean estimation & Best case estimation & Worst case estimation & Reference source \\
\hline \multicolumn{2}{|l|}{ - Nurse (20-min intervention once/year) ${ }^{\mathrm{b}}$} & $4.45 €(U S \$ 3.92)$ & $2.60 €(U S \$ 2.29)$ & $6.30 €(U S \$ 5.55)$ & c \\
\hline \multicolumn{2}{|l|}{ - Control spirometry } & 13.92€ (US \$12.26) & $13.92 €(U S \$ 12.26)$ & $13.92 €(U S \$ 12.26)$ & 7 \\
\hline TOTAL & & $25.62 €(U S \$ 22.56)$ & $20.62 €(U S \$ 18.16)$ & $30.62 €(U S \$ 26.96)$ & \\
\hline \multicolumn{6}{|l|}{ Exacerbation costs, $€(U S \$)$} \\
\hline Cost per patient & & $250 €$ (US \$1982.04) & $330 €$ (US \$290.69) & $8000 €$ (US \$7047.30) & $7,21,35$ \\
\hline
\end{tabular}

$\mathrm{CI}=$ confidence interval; $€=2017$ Portuguese euro; $\$=$ US dollar.

${ }^{a}$ Mean salary of a doctor for each hour ranges from $12,22 €$ (US \$10.76) to $31,13 €$ (US \$27.41).

${ }^{\mathrm{b}}$ Mean salary of a nurse for each hour ranges from 7,90€ (US \$6.96) to 18,80€ (US \$16.55).

${ }^{\mathrm{c}}$ Based on official values defined by the Portuguese Central Administration of Health System. 


\section{RESULTS}

\section{Cost-Effectiveness Estimation and Sensitivity Analysis}

Our analysis estimated the cost-effectiveness thresholds for intervention costs affordable limits per patient per year. Figure 2 presents these results in the range of exacerbation costs between $0 €$ (US \$0) and $8000 €$ (US \$7047.30). The respective estimation equations are as follows: (where $\mathrm{EC}=$ Exacerbation costs; IC = Intervention Costs; PIR = Probability of exacerbation under Inhaler Technique Review; and PUC $=$ Probability of exacerbation under Usual Care):

Worst case scenario: [95\% CI lower limit of $P_{U C}$ and upper limit of $\left.P_{I R}\right]$.

$$
I C=E C \times(0.54-0.47)<=>I C=0.07 \times E C .
$$

Mean estimation scenario: [mean $P_{U C}$ and mean $P_{I R}$ ]

$$
I C=E C \times(0.58-0.43)<=>I C=0.15 \times E C
$$

Best case scenario: [95\% CI upper limit of $P_{U C}$ and lower limit of $\left.P_{I R}\right]$

$$
I C=E C \times(0.62-0.40)<=>I C=0.22 \times E C
$$

In the best case scenario of intervention effectiveness and with exacerbation costs at the reported upper limit, the intervention affordable budget could be up to almost $1800 €$ (US \$1585.24) per patient per year. The more exacerbation costs increase, the higher the affordable limit rises to develop an intervention program.

\section{Cost-Effectiveness Estimations for a Portuguese Scenario}

Cost-saving estimations for Portugal were obtained considering mean values, worst and best case scenarios, and according to variations between exacerbation annual costs that range from $330.95 €$ (US \$291.54) to $8000 €$ (US \$7047.30) per patient. These reported costs concern the global population but were used here to estimate savings for older patients. The main equations used to estimate cost savings were as follows (where $\mathrm{EC}=$ Exacerbation costs; IC = Intervention costs; and RD = risk difference between Inhaler Technique Review and Usual Care):

Worst case scenario:

$$
\begin{gathered}
\text { Estimation Savings }_{\text {worst }- \text { case }}=\left(E C_{\text {best }- \text { case }} \times R D_{\text {worst } \text {-case }}\right) \\
-I C_{\text {worst } \text {-case }} \\
\text { Estimation Savings } \text { worst }- \text { case }=(330.95 \times 0.07)-30,62 \\
\text { Estimation Savings } \text { worst } \text {-case }=(-) 7,45
\end{gathered}
$$

$\underline{\text { Mean estimation: }}$

Estimation Savings $_{\text {mean }}=\left(E C_{\text {mean }} \times \mathrm{RD}_{\text {mean }}\right)-I C_{\text {mean }}$

Estimation Savings $_{\text {mean }}=(4165.48 \times 0.15)-25,62$

Estimation Savings $_{\text {mean }}=599.20$

\section{Best case scenario:}

$$
\begin{gathered}
\text { Estimation Savings }_{\text {best-case }}=\left(E C_{\text {worst-case }} \times R D_{\text {best-case }}\right) \\
-I C_{\text {best }- \text { case }}
\end{gathered}
$$

Estimation Savings worst - case $=(8000 \times 0.22)-20,62$

Estimation Savings worst - case $=1739,38$

Considering data from exacerbation risk difference reported for older patients, we estimated mean annual savings of $311.88 €$ (US \$274.68) per patient for an intervention program in Portugal. We found a wide interval between worst and best case scenarios, ranging from an annual negative balance of minus 7.45€ (US \$6.56) and a positive budget of $1739,38 €$ (US \$1532.09) per patient. However, considering the reported difference in annual costs associated with clinically well-controlled patients and clinically uncontrolled patients, which is about $469.42 €$ (US $\$ 413.47){ }^{7}$ the worst case scenario increases to a positive balance of $2.24 €$ (US \$2.13) per patient/year.

Approximately 2,2 million older adults live in Portugal. Thus considering an overall $10 \%$ combined prevalence of asthma or COPD, ${ }^{1}$ the mean estimation for effectively implemented interventions in this age group could theoretically

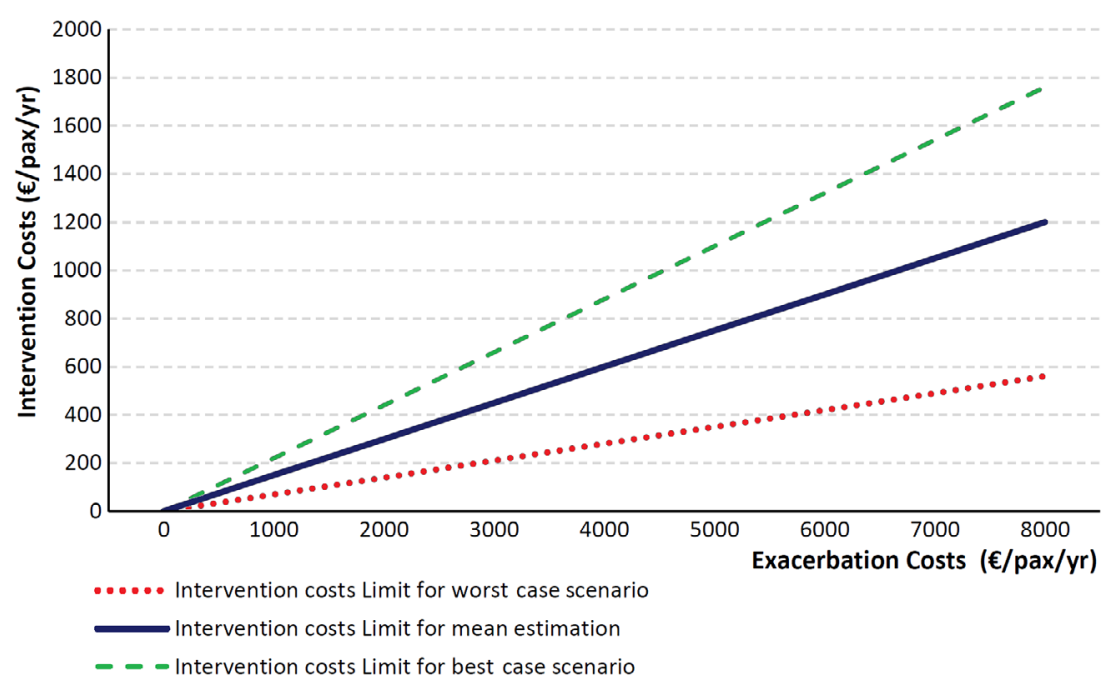

Figure 2. Cost-effectivity thresholds according to exacerbation costs. Lines represent the intervention costs affordable limit scenarios according to exacerbation proportions of $95 \%$ confidence interval (CI) lower limit, mean estimation, and $95 \%$ CI upper limit. 


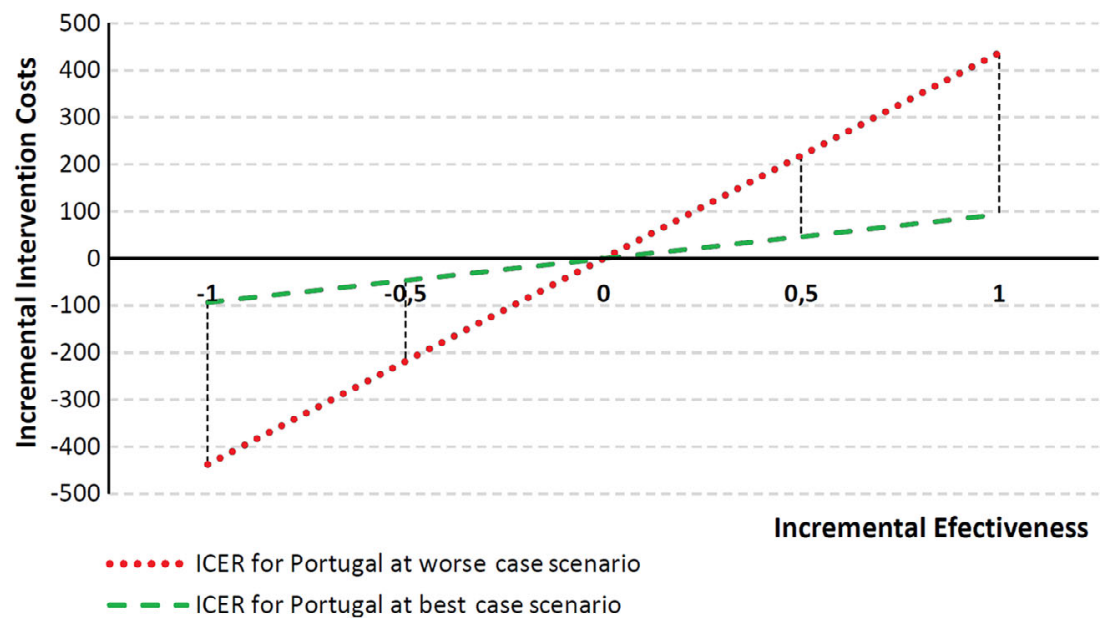

Figure 3. Incremental cost-effectiveness ratio (ICER) for Portugal at worst case and best case scenarios according to lower and upper limits of Intervention Costs and Risk Difference. Incremental effectiveness was estimated according to number of prevented exacerbations.

represent a total annual savings of $€ 131$ million (US $\$ 150$ million) for the Portuguese national health system, and this estimation already includes all the intervention costs.

\section{Incremental Cost-Effectiveness Ratio Estimations for a Portuguese Scenario}

ICER was estimated for worst and best case scenarios, and according to upper and lower limits of intervention costs and risk difference. Incremental effectiveness was estimated according to the number of prevented exacerbations. Figure $3^{31}$ represents ICER for Portugal at worst case and best case scenarios that vary between 93.73€ (US \$82.55) and $437.43 €$ (US \$385.25) per exacerbation avoided. The min equations used were as follows (where IC $=$ Intervention costs; and $\mathrm{RD}=$ risk difference between Inhaler Technique Review and Usual Care):

\section{At worst case scenario:}

$$
I C E R_{\text {worst case }}=\frac{I C_{\text {worst case }}}{R D_{\text {worst case }}}=\frac{30.62}{0.07}=437.43
$$

At best case scenario:

$$
I C E R_{\text {best case }}=\frac{I C_{\text {best case }}}{R D_{\text {best case }}}=\frac{20.62}{0.22}=93.73
$$

\section{DISCUSSION}

Our results show that interventions that include inhaler technique review to improve clinical control in older patients with asthma or COPD may be cost-effective and may generate significant savings from the perspective of the healthcare provider.

This is the first study showing that these interventions could save up to several hundreds of million euros in Portugal. Also, this is the first study establishing thresholds for affordable budget interventions that can be adopted worldwide according to exacerbations costs. Moreover, as exacerbations costs get higher, affordable limits for intervention budget also increase. These thresholds may apply to other healthcare systems because they are based on worldwide data of exacerbation rate variations and are unaffected by local health costs. In a simple approach, healthcare systems may invest in interventional programs up to $22 \%$ of total exacerbation costs to be cost-effective.

The intervention costs and exacerbation costs used were based on data from Portugal and might differ from costs in other countries that might lead to different costsaving estimations. However, when actually compared with reports from other countries, our study found similar mean savings with interventions in the Portuguese scenario, which reinforces the generalizability of our findings. In addition, most previous reports used a standard cost-effectiveness model, which was also our approach. ${ }^{15-19}$ However, similarly to these reports, we also found a very wide range of plausible values, which could be due to uncertainty of estimation of exacerbation costs and difficulty in establishing the true costs of intervention programs.

More recent studies have estimated less savings with interventions, ${ }^{15,16}$ which could be due to various reasons. First, because these studies mostly included adult patients in the base-case analysis, without focusing on older patients. Second, they mostly included COPD patients, who have irreversible airway obstruction and an increased risk of exacerbations. In asthma patients, inhaled medication, such as inhaled corticosteroids, is more effective. Finally, the observed discrepancy may also be due to different country settings and their respective costs. In contrast, we focused our analysis mainly on older patients because they tend to have higher exacerbation rates and are probably more susceptible to the benefits of interventions. ${ }^{24,25,29}$ This approach may have improved our findings. These patients also have more comorbidities and drug interactions that can increase costs of exacerbations and worsen disease control. Moreover, exacerbations may not only lead to direct costs of additional treatment and/or hospitalizations, but there is also evidence that they result in significant lung function decline and thus in a tremendous loss in overall functions, such as increased frailty and cognitive impairment. ${ }^{36,37}$

Our study has some limitations. First, we were not able to estimate and obtain indirect costs, mainly those regarding the patients' perspective, because exacerbation costs 
were uncertain in the available literature. In addition, exacerbation rates in these older patients may also be imprecise because the meta-analysis we used as the basis for our work included relatively few studies. ${ }^{29}$ Lastly, we could not perform a cost-utility analysis based on quality-adjusted life years, due to the scarcity of available data. We accept that such analysis would probably reinforce the clinical relevance of our findings.

We found that the worst case scenario for cost savings could represent a slight negative balance, but that could be underestimated. In fact, for that estimation we used data from a Portuguese study on asthma, ${ }^{7}$ but we only considered acute medical care costs that represent about $40 \%$ of patients included. However, the real prevalence of exacerbations in older patients is slightly higher. In addition, using the real difference in annual costs between controlled and uncontrolled patients (about a 469.42€ increase [US \$413.47]), our worst case scenario turns to a positive balance.

Another important aspect to consider is the intervention conceptual program itself. In fact, the mean follow-up period of previous interventional studies is wide, varying from $3^{12}$ up to 24 months, ${ }^{14}$ and none of those studies tested a regular inhaler technique review. Further studies should be designed to test how often and for how long the intervention is needed to maintain effectiveness because evidence indicates that inhaler technique review is lost after some time. ${ }^{38,39}$ Other features may also affect exacerbation risk and progressive lung function decline, such as the choice of the type of inhaler, the use of multiple devices, ${ }^{40}$ or even the choice of drugs or the combination of drugs because newer combination inhalers may make a difference in outcome. ${ }^{24,25}$

Finally, it is notable that interventions in these patients should include several aspects of disease control, such as self-management plans and inhaler technique review. This is particularly relevant because most interventional studies included in a previous meta-analysis covered other features in addition to inhaler technique review. ${ }^{29}$ In addition, most of those studies measured adherence as an outcome, rather than inhaler technique performance, an important aspect to be taken into account, because it may bias the result of intervention effectiveness. Better adherence to inhaler therapy does not necessarily mean better inhaler technique performance, which should be clarified in future studies. Also, improving adherence seems to be related to decreased exacerbation risk ${ }^{12,13}$ that may be independent of inhaler technique performance. $^{3,41,42}$ Adherence may also be an important aspect to consider in terms of cost estimation because it also affects medication costs. ${ }^{43}$ Here, medication costs were not considered as a subset, although cost estimations included them in the main source. Medication costs may decrease due to better inhaler technique because as clinical control improves, other concurrent therapies are needed less often (such as oral corticosteroids or antibiotics), and the optimal inhaled dosing may also be reduced. Those aspects were not considered in our analysis.

However, it is difficult to ascertain the cost-effectiveness of such aspects alone, mainly the impact of inhaler technique review, which was noted as a key point in previous work by others. $^{15}$

In conclusion, intervention programs in older patients with asthma or COPD seem to be cost-effective and may generate significant savings in Portugal. Also, the affordable limits for intervention costs are wide and augmented as exacerbation costs increase.

Intervention programs should embrace different dimensions such as self-management tools and inhaler technique review, which should be considered before changing or adding new treatment options.

\section{ACKNOWLEDGMENTS}

Financial Disclosure: This work was developed without any funding support or financial source. The academic affiliation of this systematic review is the Life and Health Sciences Research Institute (ICVS)/3B's at University of Minho and the Faculty of Health Sciences at the University of Beira Interior in Portugal.

Conflict of Interest: The authors declare no conflict of interest.

Authors Contributions: Study design and data extraction: Maricoto and Taborda-Barata. Data review and analysis: All authors (sensitivity analysis rechecked by MarquesGomes and Correia-de-Sousa). First draft: Maricoto. All authors commented on the first draft and agreed with the final version. Guarantors of the study: Taborda-Barata and Correia-de-Sousa.

\section{REFERENCES}

1. World Health Organization. The global burden of disease: 2004 update. 2008. http://www.who.int. Accessed September 2016.

2. Vestbo J, Anderson JA, Calverley PM, et al. Adherence to inhaled therapy, mortality and hospital admission in COPD. Thorax. 2009;64(11):939-943.

3. Simoni-Wastila L, Wei YJ, Qian J, et al. Association of chronic obstructive pulmonary disease maintenance medication adherence with all-cause hospitalization and spending in a Medicare population. Am J Geriatr Pharmacother. 2012;10(3):201-210.

4. Maricoto T, Rodrigues LV, Teixeira G, Valente C, Andrade L, Saraiva A. Assessment of inhalation technique in clinical and functional control of asthma and chronic obstructive pulmonary disease. Acta Med Port. 2015;28 (6):702-707.

5. Lavorini F, Magnan A, Dubus JC, et al. Effect of incorrect use of dry powder inhalers on management of patients with asthma and COPD. Respir Med. 2008;102(4):593-604.

6. Usmani OS, Lavorini F, Marshall J, et al. Critical inhaler errors in asthma and COPD: a systematic review of impact on health outcomes. Respir Res. 2018;19(1):10.

7. Barbosa JP, Ferreira-Magalhaes M, Sa-Sousa A, Azevedo LF, Fonseca JA. Cost of asthma in Portuguese adults: a population-based, cost-of-illness study. Rev Port Pneumol (2006). 2017;23(6):323-330.

8. Blasi F, Cesana G, Conti S, et al. The clinical and economic impact of exacerbations of chronic obstructive pulmonary disease: a cohort of hospitalized patients. PloS One. 2014;9(6):e101228.

9. Accordini S, Corsico AG, Braggion M, et al. The cost of persistent asthma in Europe: an international population-based study in adults. Int Arch Allergy Immunol. 2013;160(1):93-101.

10. Maricoto T, Madanelo S, Rodrigues L, et al. Educational interventions to improve inhaler techniques and their impact on asthma and COPD control: a pilot effectiveness-implementation trial. J Bras Pneumo. 2016;42(6): 440-443.

11. Crane MA, Jenkins CR, Goeman DP, Douglass JA. Inhaler device technique can be improved in older adults through tailored education: findings from a randomised controlled trial. NPJ Prim Care Respir Med. 2014;24:14034.

12. Tommelein E, Mehuys E, Van Hees T, et al. Effectiveness of pharmaceutical care for patients with chronic obstructive pulmonary disease (PHARMACOP): a randomized controlled trial. Br J Clin Pharmacol. 2014;77(5):756-766.

13. Khdour MR, Kidney JC, Smyth BM, McElnay JC. Clinical pharmacy-led disease and medicine management programme for patients with COPD. Br J Clin Pharmacol. 2009;68(4):588-598. 
14. Bourbeau J, Julien M, Maltais F, et al. Reduction of hospital utilization in patients with chronic obstructive pulmonary disease: a disease-specific selfmanagement intervention. Arch Intern Med. 2003;163(5):585-591.

15. Roggeri A, Micheletto C, Roggeri DP. Inhalation errors due to device switch in patients with chronic obstructive pulmonary disease and asthma: critical health and economic issues. Int J Chron Obstruct Pulmon Dis. 2016;11:597-602.

16. van Boven JF, Tommelein E, Boussery K, et al. Improving inhaler adherence in patients with chronic obstructive pulmonary disease: a cost-effectiveness analysis. Respir Res. 2014;15:66.

17. Gallefoss F, Bakke PS. Cost-effectiveness of self-management in asthmatics: a 1-yr follow-up randomized, controlled trial. Eur Respir J. 2001;17(2):206-213.

18. Tougaard L, Krone T, Sorknaes A, Ellegaard H. Economic benefits of teaching patients with chronic obstructive pulmonary disease about their illness. The PASTMA Group. Lancet. 1992;339(8808):1517-1520.

19. Bolton MB, Tilley BC, Kuder J, Reeves T, Schultz LR. The cost and effectiveness of an education program for adults who have asthma. J Gen Intern Med. 1991;6(5):401-407.

20. Van Boven J, Cushen B, Sulaiman I, et al. Personalising adherence-enhancing interventions using a smart inhaler in patients with COPD: an exploratory cost-effectiveness analysis. NPJ Prim Care Respir Med. 2018;28(1):24.

21. Direcção Geral de Saúde. Diagnóstico e tratamento da Doença Pulmonar Obstructiva Crónica. Norma número 028/2011. http://www.dgs.pt2011. [Updated 2013]:15. Accessed September 2016.

22. Organisation for Economic Co-operation and Development (OECD). Health at a glance 2017. Paris, France: OECD Publishing; 2017.

23. Barbara C, Gomes EM. Portugal - Doenças Respiratórias em Números. Programa Nacional para as Doenças Respiratórias. Direcção Geral de Saúde; 2015. http://www2.portaldasaude.pt/. Accessed May 2016.

24. Reddel H, Bacharier L, Bateman E, et al. Global strategy for asthma management and prevention. Global initiative for asthma. 2018. http://www. ginasthma.org. Accessed September 2018.

25. Vogelmeier C, Agusti A, Anzueo A, et al. Global strategy for the diagnosis, management, and prevention of chronic obstructive pulmonary disease. 2018. http://www.goldcopd.org. Accessed September 2018.

26. Allen SC, Jain M, Ragab S, Malik N. Acquisition and short-term retention of inhaler techniques require intact executive function in elderly subjects. Age Ageing. 2003;32(3):299-302.

27. Barrons R, Pegram A, Borries A. Inhaler device selection: special considerations in elderly patients with chronic obstructive pulmonary disease. Am J Health Syst Pharm. 2011;68(13):1221-1232.

28. Allen SC, Warwick-Sanders M, Baxter M. A comparison of four tests of cognition as predictors of inability to learn to use a metered dose inhaler in old age. Int J Clin Pract. 2009;63(8):1150-1153.

29. Maricoto T, Monteiro L, Gama JMR, Correia-de-Sousa J, TabordaBarata L. Inhaler technique education and exacerbation risk in older adults with asthma or chronic obstructive pulmonary disease: a meta-analysis. J Am Geriatr Soc. 2019;67(1):57-66.

30. Normansell R, Kew KM, Mathioudakis AG. Interventions to improve inhaler technique for people with asthma. Cochrane Database Syst Rev. 2017;3:CD012286.
31. Klijn SL, Hiligsmann M, Evers S, Roman-Rodriguez M, van der Molen T, van Boven JFM. Effectiveness and success factors of educational inhaler technique interventions in asthma \& COPD patients: a systematic review. NPJ Prim Care Respir Med. 2017;27(1):24.

32. Sox H, Blatt M, Higgins M, Marton K. Medical Decision Making. 2nd ed. ; Hoboken, New Jersey: Wiley-Blackwell; 2007.

33. Ryder HF, McDonough C, Tosteson ANA, Lurie JD. Decision analysis and cost-effectiveness analysis. Semin Spine Surg. 2009;21(4):216-222.

34. Husereau D, Drummond M, Petrou S, et al. Consolidated health economic evaluation reporting standards (CHEERS) - explanation and elaboration: a report of the ISPOR health economic evaluation publication guidelines good reporting practices task force. Value Health. 2013;16(2): 231-250.

35. Direcção Geral de Saúde. DPOC em números: Portugal e Europa. 2017. http://www.dgs.pt. Accessed September 2017.

36. Dodd JW, Getov SV, Jones PW. Cognitive function in COPD. Eur Respir J. 2010;35(4):913-922.

37. Villeneuve S, Pepin V, Rahayel S, et al. Mild cognitive impairment in moderate to severe COPD: a preliminary study. Chest. 2012;142(6):15161523.

38. Ronmark E, Jogi R, Lindqvist A, et al. Correct use of three powder inhalers: comparison between Diskus, Turbuhaler, and Easyhaler. J Asthma. 2005;42 (3):173-178.

39. Rootmensen GN, van Keimpema AR, Jansen HM, de Haan RJ. Predictors of incorrect inhalation technique in patients with asthma or COPD: a study using a validated videotaped scoring method. J Aerosol Med Pulm Drug Deliv. 2010;23(5):323-328.

40. Sadowski CA, Cor K, Cave A, Banh HL. Administration technique and acceptance of inhaler devices in patients with asthma or COPD. Ann Pharmacother. 2015;49(6):639-648.

41. Engelkes M, Janssens HM, de Jongste JC, Sturkenboom MC, Verhamme KM. Medication adherence and the risk of severe asthma exacerbations: a systematic review. Eur Respir J. 2015;45(2):396-407.

42. Papi A, Haughney J, Virchow JC, Roche N, Palkonen S, Price D. Inhaler devices for asthma: a call for action in a neglected field. Eur Respir J. 2011; 37(5):982-985.

43. Dekhuijzen R, Lavorini F, Usmani OS, van Boven JFM. Addressing the impact and unmet needs of nonadherence in asthma and chronic obstructive pulmonary disease: where do we go from here? J Allergy Clin Immunol Pract. 2018;6(3):785-793.

\section{SUPPORTING INFORMATION}

Additional Supporting Information may be found in the online version of this article.

\section{Appendix S1. CHEERS recommendations checklist}

